\title{
Space-Time Array Communications
}

Vector Channel Estimation and Reception 
This page intentionally left blank 


\section{Space-Time Array Communications}

Vector Channel Estimation and Reception

\section{Jason W. P. Ng}

British Telecommunications Research Labs, UK

Imperial College Press 


\section{Published by}

Imperial College Press

57 Shelton Street

Covent Garden

London WC2H 9HE

\section{Distributed by}

World Scientific Publishing Co. Pte. Ltd.

5 Toh Tuck Link, Singapore 596224

USA office: 27 Warren Street, Suite 401-402, Hackensack, NJ 07601

UK office: 57 Shelton Street, Covent Garden, London WC2H 9HE

\section{British Library Cataloguing-in-Publication Data}

A catalogue record for this book is available from the British Library.

\section{SPACE-TIME ARRAY COMMUNICATIONS \\ Vector Channel Estimation and Reception}

Copyright () 2007 by Imperial College Press

All rights reserved. This book, or parts thereof, may not be reproduced in any form or by any means, electronic or mechanical, including photocopying, recording or any information storage and retrieval system now known or to be invented, without written permission from the Publisher.

For photocopying of material in this volume, please pay a copying fee through the Copyright Clearance Center, Inc., 222 Rosewood Drive, Danvers, MA 01923, USA. In this case permission to photocopy is not required from the publisher.

ISBN-13 978-1-86094-672-1

ISBN-10 1-86094-672-0

Printed in Singapore. 


\section{To my wife Janice and my son Vincent}


This page intentionally left blank 


\section{Preface}

Space-time array communications - vector channel estimation and reception is stemmed from the integration of two emerging technologies for the future wireless mobile systems: space-time array processing and spread spectrum multiple access communications. The vector channel constitutes the spatial-temporal multipath propagation environment resulting from multiple reflection, refraction, diffraction and scattering phenomena experienced by the propagating signal. By applying spacetime processing in spread spectrum multiple access system, the multiuser vector channel can be exploited, in accordance to its environmental context, to provide a mitigation of the various associated channel impairments. As a consequence, new dimensional layer of interference cancellation methodology can be devised, and additional form of inherent diversity can be derived, based on specifically developed vector channel estimation technique integrated at the front-end of the reception process. This book will look at the following three original integrated vector channel estimation and reception algorithms, with each dealing with different practical environmental scenario.

First is the Polarisation-Space-Time estimation and reception. Traditional array processing techniques often ignore the polarisation aspect of the signal, disregarding the fact that, in typical mobile environment, the received signal rarely takes on its transmitted polarisation. The diversity in the signal polarisation is not insignificant due to the depolarisation mechanism intrinsical in the propagation channel, and considering the recent development of portable handheld terminals which are often randomly orientated. Hence, instead of taking the polarisation factor as part of signal fading, the polarisation information inherent in the signal can be exploited to improve the accuracy and resolution of the estimation process and enhance the detection capability of the receiver.

Second is the Diffuse-Space-Time estimation and reception. Most array processing models frequently assume a rather simplistic view of 
the multipath propagation environment, which is made up of multiple point sources in the channel. However in typical wireless urban or suburban setup, the signal transmitted into the channel may suffer localised scattering which will inevitably create diffusion in its signal components. This consequently leads to a performance degradation when conventional array processing technique, founded on point sources assumption, is employed. A generalised diffusion framework is therefore devised to cope with both point and/or diffuse sources resulted in the signal environment. Its underlying architecture also permits ease in an extension to a co-code environmental scenario.

Third is the Doppler-Space-Time estimation and reception. Doppler spread, due to the relative motion in the environment, is detrimental in typical Multiple Input Multiple Output (MIMO) system. This can however be incorporated as a source of diversity by exploiting these multipath Doppler components in the receiver design. Unlike many MIMO works which are based on multiple independent antenna elements, the proposed MIMO array receiver is built on antenna array technology which is commonly used in applications such as the wellknown smart-antenna system. It does not require the imposition of the knowledge of the channel or the need of any power control as in its conventional counterparts.

To gain a deeper understanding in the above algorithmic frameworks, other representative examples are also included at the end of the text to allow new insights into the application of these algorithms. Following that, some of the key points in the current research are highlighted, which offer some indicative views into the various potential areas for further investigative studies.

Note that this book is meant to provide a holistic picture in the treatment of the subject, bringing and linking together a number of the author's main relevant publications. It is hopeful that the readers will find the text useful, in not only gaining a comprehensive understanding but also in extending the work to other fields of research. 


\section{Contents}

Chapter 1: Introduction

1.1 Spread Spectrum Multiple Access Scheme ……................................ 3

$1.2 \quad$ Antenna Array Communications ...................................................... 7

1.2.1 Wireless Multipath Channel Characterisation ........................ 8

1.2.2 Space-Time Array Processing ............................................ 12

$1.3 \quad$ Motivation and Organisation of Book ………................................. 16

Chapter 2: $\quad$ Spatial-Temporal ARray (STAR) Architecture

2.1 Basic Elements of System Model ………...................................... 20

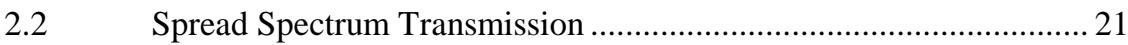

2.3 Multiuser Space-Time Vector Channel ............................................. 23

2.3.1 Sensor Array Manifold Vector ............................................ 23

2.3.2 Vector Channel Mathematical Model ................................... 24

2.3.3 Continuous-Time Signal Model ........................................... 25

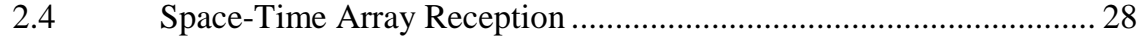

2.4.1 Front-End Temporal Windowing ........................................ 28

2.4.2 Spatial-Temporal Array Manifold Vector ............................ 30

2.4.3 Discrete-Time Signal Model .............................................. 32

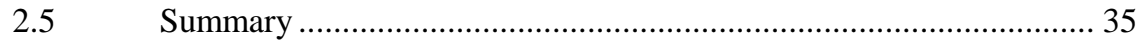


Chapter 3: $\quad$ Polarisation-Space-Time Estimation and Reception

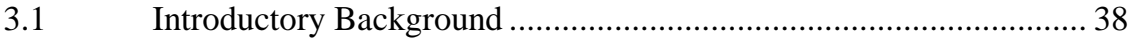

3.2 Polarised Array Manifold Vector ......................................................... 40

3.2.1 Polarisation of Electromagnetic Wave ................................ 40

3.2.2 Polarisation-Sensitive Array Response ................................. 43

3.2.3 Diversely-Polarised Manifold Shape .................................... 46

3.3 Polar-STAR Signal Modelling .................................................... 47

3.4 Vector Channel Estimation and Reception ...................................... 49

3.4.1 Operation of Preprocessor .................................................... 49

3.4.2 Spatial-Temporal Smoothing Technique ............................. 51

3.4.3 Polarisation-Angle-Delay Estimation (PADE) Algorithm ... 54

3.4.4 Polarisation-Space-Time Reception .................................... 57

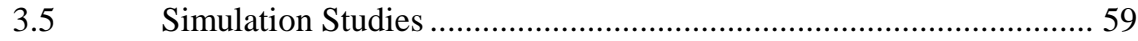

3.5.1 Performance of Polarisation-Space-Time Algorithm ........... 59

3.5.2 Studies of Closely-Located Paths ......................................... 64

3.5.3 Exemplification of Ill-Conditioned Event ......................... 67

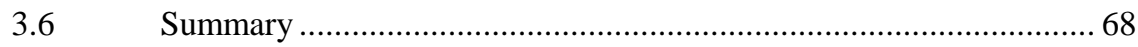

Chapter 4: $\quad$ Diffuse-Space-Time Estimation and Reception 69

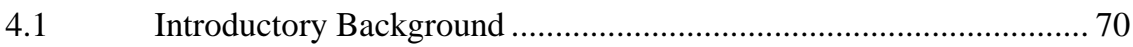

4.2 Diffuse Array Manifold Vector ........................................................ 72

4.3 Diffuse-STAR Signal Modelling ……………….......................... 75

4.4 Vector Channel Estimation and Reception ....................................... 76

4.4.1 Blind Diffuse-Space-Time Estimation .................................. 76

4.4.2 Co-code/Non Co-code Diffusion-Based Reception .............. 80

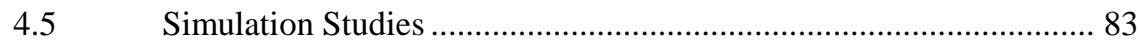

4.5.1 Performance of Diffuse-Space-Time Algorithm .................. 83 
4.5.2 Robustness to Channel Estimation Errors ………………..... 87

4.5.3 Evaluation of Diffusion Framework Reception ................... 89

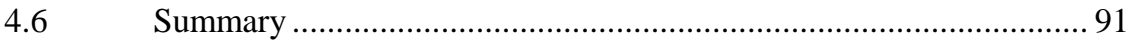

Chapter 5: $\quad$ Doppler-Space-Time Estimation and Reception

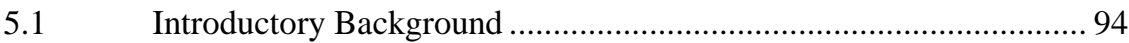

5.2 MIMO Array System Formulation ………...................................... 96

5.2.1 Continuous Time-Varying Signal Model ............................. 96

5.2.2 Discrete Time Doppler-STAR Signal Model ...................... 98

5.3 Vector Channel Estimation and Reception ..................................... 100

5.3.1 Joint Space-Time Channel Estimation ................................ 100

5.3.2 MUSIC/Analytical Approach to Doppler Estimation ......... 101

5.3.3 Robust MIMO Array Reception .......................................... 103

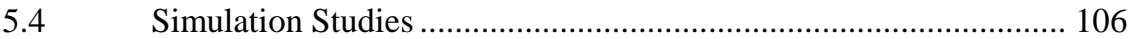

5.4.1 Performance of Doppler-Space-Time Algorithm .............. 106

5.4.2 Comparison of Doppler Estimation Approaches ................ 110

5.4.3 Investigation of Near-Far Resistant Capability .................. 112

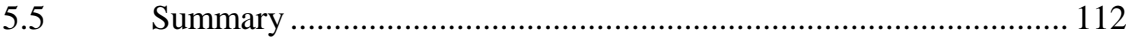

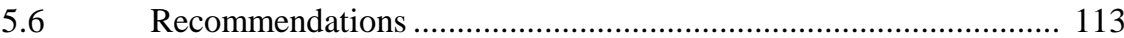

Chapter 6: $\quad$ Estimation and Reception Application Examples

6.1 SIMO Sensor Network Array System .............................................. 116

6.1.1 SIMO Sensor Network Signal Model ................................ 116

6.1.2 Blind Estimation and Reception ...................................... 118

6.1.3 Performance Analysis ....................................................... 121

6.1.4 Concluding Summary ................................................... 124 
6.2 MIMO OTD Array System ........................................................... 125

6.2.1 MIMO OTD Signal Model ................................................ 125

6.2.2 Blind Estimation and Reception ......................................... 128

6.2.3 Performance Analysis ........................................................ 129

6.2.4 Concluding Summary ........................................................ 131

6.3 MIMO Diversely Polarised Array System …………………........... 132

6.3.1 MIMO Diversely Polarised Signal Model ......................... 132

6.3.2 Blind Estimation and Reception ....................................... 134

6.3.3 Performance Analysis ..................................................... 135

6.3.4 Concluding Summary …............................................... 137

Chapter 7: $\quad$ Discussions on Present and Future Research Work 139

7.1 Key Points of Current Work ……………................................... 139

7.2 Directions for Further Research ................................................... 143

$\begin{array}{ll}\text { Abbreviations \& Notations } & 147\end{array}$

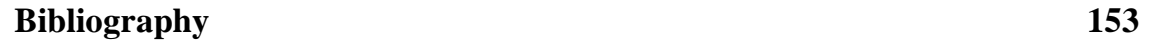

